\title{
Mental Health Symptoms in Children and Adolescents during COVID-19 in Australia
}

Sicouri $\mathrm{G}^{1}$, March $\mathrm{S}^{2}$, Pellicano $\mathrm{E}^{3}$, De Young AC $\mathrm{A}^{4,6}$, Donovan, $\mathrm{CL}^{5}$, Cobham, $\mathrm{VE}^{6,7}$, Rowe $\mathrm{A}^{9}$, Brett, $\mathrm{S}^{3,8}$, Russell $\mathrm{JK}^{9}$, Uhlman, $\mathrm{LR}^{7}$, \& Hudson, $\mathrm{JL}^{1}$

${ }^{1}$ Black Dog Institute, University of New South Wales, Sydney, NSW, Australia.

${ }^{2}$ School of Psychology and Counselling and Centre for Health Research, University of Southern Queensland, Springfield, Queensland, Australia.

${ }^{3}$ Macquarie School of Education, Macquarie University, Sydney, New South Wales, Australia.

${ }^{4}$ Children's Health Research Centre, The University of Queensland, Brisbane, Queensland, Australia.

${ }^{5}$ School of Applied Psychology, Griffith University, Queensland, Australia.

${ }^{6}$ School of Psychology, The University of Queensland, Saint Lucia, Queensland, Australia.

${ }^{7}$ Children's Health Queensland, Child and Youth Mental Health Service, Brisbane,

Queensland, Australia

${ }^{8}$ University of Reading, Reading, United Kingdom.

${ }^{9}$ Centre for Health Research, University of Southern Queensland, Queensland, Australia.

Corresponding author at: Black Dog Institute, University of New South Wales, Hospital Rd, Randwick, NSW 2031, Australia. Email address: g.sicouri@blackdog.org.au (G. Sicouri). 


\begin{abstract}
Objective: COVID-19 has led to disruptions to the lives of Australian families through social distancing, school closures, a temporary move to home-based online learning, and effective lockdown. Understanding the effects on youth mental health is crucial to inform policies to support communities as they face the pandemic and future crises. This paper sought to report on mental health symptoms in Australian children and adolescents during the initial stages of the pandemic (May to November 2020) and to examine its association with child/family characteristics and exposure to the broad COVID-19 environment. Methods: An online longitudinal survey was completed by 1,324 parents and carers of Australian children aged 4 to 17 years. Parents/carers reported on their child's mental health using five measures, including emotional symptoms, conduct disorder problems, hyperactivity/inattention, anxiety symptoms and depressive symptoms. Child/family characteristics and COVID-related variables were measured. Results: Overall, $30.5 \%, 26.3 \%$ and $9.5 \%$ of our sample scored in the high to very high range for emotional problems, conduct problems and hyperactivity/inattention, respectively. Similarly, $20.2 \%$ and $20.4 \%$ of our sample scored in the clinical range for anxiety symptoms and depressive symptoms respectively. A child's preexisting mental health diagnosis, neurodevelopmental condition and chronic illness significantly predicted parent-reported youth mental health symptoms. Parental mental health symptoms, having a close contact with COVID-19 and applying for government financial assistance during COVID-19 were also significant predictors of youth mental health symptoms. Conclusions: Our findings show that Australian youth experienced considerable levels of mental health symptoms during the initial phase of COVID-19, and highlight the need for targeted, effective support for affected youth and particularly for those with preexisting vulnerabilities. Keywords: adolescent, children, COVID-19, pandemic, mental health, anxiety, depression, Australia
\end{abstract}




\section{Mental Health Symptoms in Children and Adolescents during COVID-19 in Australia}

In Australia, as of $3^{\text {rd }}$ June 2021, there have been 30,118 confirmed cases and 910 deaths, placing it in the lowest quartile of infection rates and deaths per capita (WHO, 2021). Australia's low rate of infection is partly explained by the federal and state governments use of increasingly restrictive measures to reduce the infection rate. These restrictions have led to disruptions to Australian families lives through social distancing, school closures and a temporary move to home-based online learning, and effective lockdowns. As COVID-19 and the restrictions continue, there is a growing concern about the impact on mental health - and youth mental health in particular (Holmes et al., 2020). Few studies have determined the impact of pandemic-related disruptions on children and adolescents, which is worrying, given the importance of peer interaction and social connection in these development stages (Orben et al., 2020). Understanding the mental health effects of the COVID-19 pandemic on children and adolescents, in the context of known and pandemic-related risk factors, is crucial to inform how policymakers should best support communities as they face the ongoing COVID19 pandemic and future crises.

The limited research that does exist has shown that children and young people have experienced high levels of mental health symptoms during the pandemic (Racine et al., 2020). For example, a study in China found that $18.9 \%$ of children (in grades $2-6$ ) reported elevated anxiety symptoms and $22.6 \%$ of children reported elevated depressive symptoms (Xie et al., 2020). A large cohort study $(n=12,500)$ tracking changes in youth mental health since early lockdown in the UK, named Co-SPACE (COVID-19: Supporting Parents, Adolescents and Children during Epidemics) (Waite \& Creswell, 2020) found a rise in parent-reported child and adolescent mental health symptoms in the early lockdown period (March-June 2020), with hyperactivity/inattention and conduct problems increasing. Emotional symptoms increased in preadolescents, but slightly decreased for adolescents 
(Waite et al., 2021). Another non-peer-reviewed study (predominantly US) found that selfreported depressive symptoms, but not anxiety symptoms, had increased among adolescents over the first six months of the pandemic (Barendse et al., 2021). Together, these studies show that child and adolescent mental health symptoms have worsened during the pandemic.

In Australia, there is emerging evidence that youth mental health is also deteriorating. Magson et al. (2020) found that self-reported depressive and anxiety symptoms in 13- to 16year-old Australians, increased from before the COVID-19 outbreak to two months following government restrictions in May 2020, and that this increase was particularly pronounced in females. Li et al. (2021), showed that 75\% of adolescents (aged 12-18 years) reported worsening mental health since the pandemic began (to August 2020), and that these effects were more pronounced among adolescents with a pre-existing anxiety or depression diagnosis. A non-peer reviewed study reported that parent-reported child depressive symptoms did not increase compared to pre-pandemic estimates (Westrupp et al., 2021). Beyond these studies, estimates of mental health symptoms in children and adolescents particularly those reported by parents - during the pandemic in Australia is unknown, and no studies report on the magnitude or clinical significance of problems. Given the severity of restrictions in Australia and the relatively high degree of compliance by Australian citizens (Pfeffer, 2021), as well as an increase in help-seeking (Young, 2020), it is likely that Australian youth mental health may have fared particularly badly.

Pandemic aside, risk factors known to be associated with poor youth mental health generally include coming from a low income (Wickham et al., 2017) or single parent household (Nguyen et al., 2017), being Aboriginal or Torres Strait Islander (Zubrick et al., 2005), having a neurodevelopmental condition or special need (Hansen et al., 2018), having a chronic physical health condition (Pinquart and Shen, 2011a; 2011b), or having a parent with a mental health disorder (Lawrence et al., 2019). There are also differences across gender and 
age with respect to risk for youth mental health problems. Males are more likely to have any mental health disorder across childhood and adolescence $(16.3 \%$, most commonly behavioural problems) compared to females (11.5\%), although during adolescence, females are more likely to experience anxiety or depression $(7.7 \%$ and $5.8 \%$ respectively) than males (6.3 and 4.3\% respectively; Lawrence et al., 2015). Furthermore, younger children may have faced particular challenges given they are less likely to learn independently while out of school and are more dependent on their parents (who have experienced high levels of stress during the pandemic; Whittle et al., 2020). However, adolescents are likely to have faced challenges given their developmental need for autonomy and social connection, which have been disrupted by restrictions. Research from the Co-SPACE UK study has shown that a child's neurodevelopmental condition, parent/carer mental health symptoms and younger age, were associated with poorer trajectories of mental health symptoms (Raw et al., 2021).

In addition to known risk factors, it is important to investigate the relationship between mental health symptoms and risk factors associated with COVID-19, such as exposure to COVID-19, and impacts on parental employment and household finances, given that loss of employment and financial strain are well-documented correlates of youth mental health problems (Bubonya et al., 2017). In Australia, social distancing measures and lockdown resulted in increases in unemployment, causing financial difficulties for many households. For youth, social isolation and loneliness as a result of restrictions is also likely to be a key factor associated with anxiety and depressive symptoms (Loades et al., 2020), and the longer the pandemic and associated restrictions continue, it may be that youth mental health symptoms deteriorate further (Raw et al., 2020). The degree and nature of restrictions have varied across the states of Australia and during the course of the pandemic. Given that stricter government restrictions were negatively associated with adolescents' mental health 
symptoms outside of Australia (Barendse et al., 2021), it seems possible that the severity and nature of restrictions in Australia would be associated with youth mental health.

The aim of the current study was to assess the impact of the COVID-19 pandemic in Australia on young people's mental health. We extended the Co-SPACE UK research to an Australian context, and report on broad measures of parent-reported mental health symptoms, namely, the three subscale measures of the Strengths and Difficulties Questionnaire (Goodman, 1997), measuring emotional symptoms, conduct disorder problems and hyperactivity/inattention. We also report on outcomes related to youth anxiety symptoms and depression symptoms separately, as the emotional symptom subscale includes items related to both anxiety and depression, which may mask differences in outcomes for these symptom domains. The first aim was to report on mental health symptoms in a sample of children and adolescents during the COVID-19 pandemic in Australia. The second aim was to examine associations between known risk factors (child/family characteristics) and COVID-19 specific risk factors (e.g., exposure to COVID-19) and youth mental health symptoms.

\section{Method}

\section{COVID-19 in Australia}

In Australia, the number of confirmed COVID-19 cases was over 1,000 on $21^{\text {st }}$ March 2020, ten days after the World Health Organisation declared the outbreak a pandemic. On this date, Australian states and territories, with the support of the federal government, began to implement a strategy known as "aggressive suppression" of the virus (Bromfield \& McConnell, 2020). Non-essential services, including restaurants and sporting venues were forced to close; domestic and international borders were closed; schools began to close in 
most territories and states, and strict limits were placed on social gatherings. Many of these restrictive measures began to ease at the end of April 2020, with schools opening again for a significant majority of children by $27^{\text {th }}$ May 2020. In the subsequent five months, there were varying stages of lockdown in different states and territories, including a second lockdown in Melbourne and Victoria. At the end of the recruitment period (16 ${ }^{\text {th }}$ November, 2020), there had been over 55 million COVID-19 cases and 1.3 million COVID-related deaths worldwide, with 27,725 cases and 907 COVID-related deaths in Australia.

\section{Study Design and Participants}

Data were collected via two longitudinal surveys established on the Limesurvey and Qualtrics survey platforms. The surveys were set up in two states of Australia (NSW and Queensland) to capture a broader geographical sample (although participants from all states could participate in both surveys). The surveys included the same questionnaires but differed slightly to capture each research groups' area of interest and issues specific to the region. Parents $^{1}$ (aged 18 years and over) of school-aged children and adolescents (aged $4-17$ years) who lived in Australia were eligible to take part. During the second lockdown in Victoria, we targeted parents living in Victoria specifically to include participants who experienced the highest level of government restrictions. A total of 1,324 participants were recruited through advertisements on social media $(n=403)$ or via a panel provider, CINT $(n=924)$.

Participants recruited through a panel provider were initially screened to determine eligibility and were reimbursed between $\mathrm{A} \$ 5$ and $\mathrm{A} \$ 6$. Participants recruited via social media were given the option of entering a draw to win one of three $\mathrm{A} \$ 40$ vouchers. We report data on 1,397 participants who completed the baseline survey online between $5^{\text {th }}$ May and $16^{\text {th }}$

${ }^{1}$ The term "parent" will refer to the wide range of people in a primary caregiving role in relation to a child or adolescent. 
November 2020. The self-selecting nature of the recruitment means that this is not a nationally representative sample. Participant demographic information is shown in Table 1.

\section{Procedure}

Parents provided written informed consent and then completed the survey online. If participants had more than one child, they were asked to choose one "index" child on which to report. Research ethics approval was obtained from the participating Universities Human Research Ethics Committees (Griffiths University: 2020/350; Macquarie University: 52020663515639; University of Southern Queensland: H20REA112; University of Queensland: H2OREA112).

\section{Measures}

\section{Child and family characteristics}

Parents reported on their own and their child's age, gender, ethnicity, Aboriginal and Torres Strait Islander origin, geographic state, postcode and household income. A household income of less than $\$ 41,599$ was categorised as low income as it is $55 \%$ less than the Australian median household income of $\$ 95,732$ per year. Parents were asked about their relationship status, their relationship to their child, the child's biological relationship to the parent, their education status, their child's education type, their employment status before COVID-19 and whether it had changed during COVID-19. Parents were asked whether they had received government financial assistance before COVID-19 and whether they had applied for government financial assistance during COVID-19. They were asked whether their child had a pre-existing and diagnosed neurodevelopmental condition (e.g., autism), mental health disorder (e.g., anxiety) or chronic health condition (e.g., asthma). Parents rated their child's 
overall mental health prior to COVID-19 (five-point Likert-type rating, $1=$ poor to $5=$ excellent).

Strengths and Difficulties Questionnaire - Parent report (SDQ; Goodman, 1997)

The parent-report version of the SDQ was used to measure youth mental health symptoms. The SDQ has satisfactory psychometric properties across the study age range (Stone et al., $2010)$ and consists of 25 items, each rated on a 3 -point Likert scale $(0=$ not at all; $2=$ certainly true). The items load on to five subscales, each with five items. In the current study, we examined the three subscales related to mental health symptoms, namely emotional symptoms, conduct problems and hyperactivity/inattention. Subscale scores were calculated by summing the responses of items from each subscale (range $0-10$ ). If there was missing data on subscale items, the mean was imputed if at least $60 \%$ of subscale items were completed. We calculated elevated levels of symptoms on each of the subscales using cut-off scores (Goodman \& Goodman, 2009): slightly raised (80 - 90 ${ }^{\text {th }}$ percentile) and high to very high $\left(>90^{\text {th }}\right.$ percentile) levels of symptoms. The Cronbach's alpha estimates for the current sample were acceptable $(\alpha=.83, \alpha=.71$ and $\alpha=.69$ for emotional symptoms, conduct problems and hyperactivity/inattention, respectively).

Revised Children's Anxiety and Depression Scale 25 - Parent version (RCADS25-P; Ebesutani et al., 2016)

The RCADS25-P assesses parent-report of youth anxiety and depression symptoms and has demonstrated satisfactory psychometric properties for children in Years 3 to 12 (aged 8 to 17 years). Given the scale has not been validated for children aged 4 to 7 we conducted a subanalysis comparing the psychometric properties of the scale for these children compared to the rest of the sample (see Table 7, Supplementary Materials). The results were similar across 
age groups, so we report on outcomes for the total sample. The scale consists of 25 items, each rated on a 4-point Likert scale ( $0=$ never; 3 = always). Two subscale scores relating to anxiety symptoms (15 items; range $0-45)$ and depressive symptoms (10 items; range $0-30$ ) are calculated by summing responses of the subscale items. If there were missing data, the person mean was imputed on responses if there were less than or equal to 2 response items missing on each of the subscales. The subscales were adjusted for the child's grade and gender to create normalised t-scores, which were used to determine the level of symptom severity (t-score: $65-70=$ borderline; 70+ = elevated). Cronbach's alpha estimates for the current study were high $(\alpha=.93$ and $\alpha=.92$ for the anxiety symptom and depressive symptom subscales, respectively).

Depression Anxiety and Stress Scales (DASS21; Lovibond and Lovibond, 1995)

The DASS-21 was used to measure total parental mental health symptoms over the past two weeks. Twenty-one items were scored on a 3-point Likert scale $(0=$ Did not apply to me at all; 3 = Applied to me very much, or most of the time). Items were summed and doubled to yield a total score (maximum score $=126$ ), with lower scores indicating lower levels of symptoms. The DASS-21 has demonstrated satisfactory psychometric properties (Lovibond \& Lovibond, 1995) and internal consistency for the current sample was high $(\alpha=.97)$.

\section{COVID-19 Exposure}

Participants were asked, "Has your child had COVID-19?” with four response options (No/Suspected/Yes, diagnosed and recovered/Yes, diagnosed and still ill), which were dichotomised for analysis purposes (No/Yes (suspected or diagnosed). The COVID-19 status of the child's close contacts was assessed, "Has anyone close to your child been diagnosed with COVID-19" (Yes/No). Parents were also asked, "How much is your child asking 
questions, reading or talking about COVID-19?" (5-point Likert-type rating, 1 = never; $5=$ most of the time).

\section{Self-isolation Status}

Self-isolation status was assessed using eleven response options, including: "I am living my life as normal" to "I am self-isolating due to a diagnosis or COVID-19 possible symptoms" (see Table 5, Supplementary Material). We dichotomised responses to reflect the degree of current isolation status, namely " $0=\mathrm{I}$ am living life normally/I am not strictly self-isolating” versus " 1 = I am strictly self-isolating" (e.g., not leaving home at all).

\section{Stage of lockdown/Time since onset of the pandemic}

Two variables were created to account for (1) the stage of lockdown at the time of survey completion; and (2) the time since onset of the pandemic. The stage of lockdown variable measured the severity of restrictions in place at the time of survey completion and area postcode, ranging from stage 1 (few to no restrictions) to stage 5 (enforced stay-at-home orders). Stages were created by the study authors based on classification systems in Australia (Table 6, Supplementary Materials). The time since onset of the pandemic variable was created by calculating the number of days from the start of the pandemic (i.e., $11^{\text {th }}$ March, $2020)$ to the date of survey completion ( $M=126$ days, $S D=49.2$ days, range $=63-250$ days).

\section{Data Analysis}

Our first aim was addressed by reporting on child and family characteristics using descriptive analyses. We dichotomised age to create two variables to reflect the typical patterns of child and adolescent mental health and the different education experiences 
$($ children $=$ aged 4 to 11 years; adolescents $=$ aged 12 to 17 years). We report on outcomes for each age group, as well as the total sample. We report on five primary outcome variables related to mental health: SDQ emotional symptoms, SDQ conduct disorder problems, SDQ hyperactivity/inattention, RCADS25-P anxiety symptoms and RCADS25-P depressive symptoms. We used published cut-off scores to determine the proportion of our sample who experienced different category thresholds of symptoms. No cut off scores were available for children in grades $\mathrm{K}$ to Year 2 for RCADS25-P, so we used the cut off scores for the lowest grade available (i.e., Year 3).

Our second aim was addressed using raw scores for our five outcome measures. First, initial associations between each outcome variable and each predictor variable were examined using univariate analyses. If a predictor variable was significantly associated with the outcome variable in the univariate analysis, it was included in the multiple linear regression model for that outcome variable. Multiple linear regression was used to investigate relationships between predictor variables and each of the outcome measures, controlling for other measures in the model. Twenty-nine predictor variables were assessed and grouped: child/family characteristics (21 variables; split into child/parent demographics (12), child clinical/health background (3), family factors/functioning (3), relationship factors (3); COVID-19 exposure variables (2); COVID-19 impact/measures (6). Predictor variables with more than two categories were collapsed into two categories and dummy coded. Models were checked and showed an absence of multicollinearity, outliers and non-normality of residuals. In accordance with Perneger (1998), we report outcomes without adjusting for multiple comparisons. Analyses were conducted in SPSS version 27.

\section{Missing data}

Data for participants were included in the analyses when a participant had completed one of the primary outcome measures. Overall, $<1 \%$ of the data were missing with the exception of 
the variables measuring government financial assistance before or during COVID-19 ( $\mathrm{n}=39$, $2.9 \% ; \mathrm{n}=117,8.8 \%$ missing responses for each variable respectively). Both had a "not applicable option" for participants who were not eligible to apply. Due to minimal missing data, all analyses were conducted using listwise deletion.

\section{Results}

\section{Prevalence estimates and clinical characteristics}

Overall, $30.5 \%, 26.3 \%$ and $9.5 \%$ of our sample scored in the high to very high range for emotional problems, conduct problems and hyperactivity/inattention, respectively. For anxiety and depressive symptoms, $20.2 \%$ and $20.4 \%$ of our sample respectively scored in the clinical range. Prevalence estimates were 2 to 3 times higher in children and adolescents with a pre-existing mental health diagnosis. Children experienced higher rates of high to very high levels of emotional symptoms, conduct problems and hyperactivity/inattention, than adolescents. On anxiety and depressive subscales, clinical levels of anxiety symptoms and depressive symptoms were similar across age groups, with approximately 1 in 5 children or adolescents experiencing clinical levels of symptoms. Of those children and adolescents whose mental health was rated as very good or excellent prior to COVID-19, 23.8\%, 23.2\% and $7.7 \%$ scored in the high-very high range for emotional problems, conduct problems and hyperactivity/inattention, respectively at the time of survey completion. Similarly, $16.4 \%$ and $16.0 \%$ of children who were rated as having very good or excellent mental health prior to the pandemic, scored in the clinical range for anxiety symptoms and depressive symptoms at the time of survey completion, respectively (see Table 2 for prevalence estimates).

\section{Univariate Relationships}

Univariate outcomes are shown in Tables 8 and 9, Supplementary Material. 


\section{Multiple Linear Regression}

The predictor variables accounted for a significant amount of variance in each model $\left(R^{2}\right.$ range $=.36-.52, p<.001$; see Tables 3 and 4 for regression results).

\section{Child and family characteristics}

\section{Demographics}

Parents who were younger and male reported higher levels of conduct problems and anxiety symptoms in their child. Younger children were reported to have higher levels of conduct problems and hyperactivity/inattention compared to older children. Females were reported to have higher levels of emotional symptoms and anxiety symptoms compared to males, whereas males were reported to have higher levels of hyperactivity/inattention by their parents.

\section{Child clinical/health background}

A child's pre-existing mental health diagnosis was a significant predictor of parent reported child mental health symptoms across all five outcome measures. A child's neurodevelopmental condition was a significant predictor of parent reported mental health symptoms for four of the five outcome measures: conduct problems, hyperactivity/inattention, anxiety symptoms and depressive symptoms. Children with a chronic illness were reported to have higher levels of anxiety and depressive symptoms.

\section{Family factors/functioning}

Parents' mental health symptoms were a significant predictor of parent reported youth mental health symptoms across all five outcome measures. 


\section{COVID-19 exposure}

If a child had a close contact who was diagnosed or suspected of having COVID-19, their parents reported the child as having higher levels of conduct problems, anxiety symptoms and depressive symptoms.

\section{COVID-19 impact and restrictions}

Application for government financial assistance during COVID-19 significantly predicted parent reported emotional symptoms and depressive symptoms. Children asking more frequent questions about COVID-19 had higher levels of emotional symptoms, anxiety symptoms and depressive symptoms. Time since onset of the pandemic was a significant predictor of parent report of child conduct problems. The longer the pandemic lasted, the higher levels of conduct disorder problems reported.

\section{Discussion}

The current study reports - for the first time to our knowledge - the prevalence of parentreported mental health symptoms in children and adolescents during the initial stages of the COVID-19 pandemic in Australia. The results showed that approximately one in three children and adolescents reportedly experienced high to very high levels of emotional symptoms, one in four experienced high to very high levels of conduct disorder problems and one in 10 experienced high to very high levels of hyperactivity/inattention. Similarly, Results indicated that approximately one in five children and adolescents reportedly experienced symptoms of anxiety and/or depression indicative of a mental health disorder. We do not have pre-pandemic data so we cannot comment on changes in the prevalence of mental health problems because of the pandemic. Nevertheless, these rates are high compared to pre- 
pandemic levels in normative, general population data (e.g., Lawrence, 2015), although comparisons are highly tentative due to the non-representative sample and symptom measures used in the current study. Mean level of emotional symptoms, conduct disorder problems and hyperactivity/inattention were between $1.2 \mathrm{x}$ and $1.7 \mathrm{x}$ higher compared to a study using the same measures in a non-representative child sample pre-pandemic (Seward et al., 2018). Further, mean levels of symptoms for the three SDQ subscales were comparable to those reported in UK children and adolescents during a similar time period (Pearcey et al., 2020). We do not have comparison data for the RCADS25-P. Overall, these findings indicate that the prevalence and mean levels of parent-reported youth mental health symptoms are notably high - and comparable to UK data in a similar aged sample - during the initial stages of the pandemic in Australia.

The findings indicate that parent-reported youth mental health symptoms have deteriorated during the pandemic. Approximately $23 \%$ of parents who reported their child as having excellent or very good mental health prior to the pandemic reported high to very high scores for emotional symptoms and conduct disorder problems. Similarly, around $16 \%$ of parents who reported their child as having excellent or very good mental health prior to the pandemic reported clinical levels of anxiety and depressive symptoms in their child. We need to acknowledge that obtaining pre-pandemic retrospective estimates of mental health from parents without the use of validated symptoms measures is a limitation of the current research. Further, while our pattern of findings is consistent with other studies that have examined adolescent self-reported symptoms (Magson et al., 2020, Li et al., 2021), we also consider the possibility that parents may not be fully aware of the extent of their child's symptoms, particularly emotional, anxiety or depressive symptoms (Salbach-Andrae et al., 2009) and thus, our results may be an underestimate of these difficulties. Nevertheless, our 
results support other research that youth mental health symptoms have deteriorated over the course of the pandemic.

There were few differences in the extent of symptoms over time according to child and family characteristics, with a few exceptions. For example, parents reported higher levels of conduct disorder problems and hyperactivity/inattention in younger children. However, child's age was not a significant predictor of emotional symptoms, anxiety or depressive symptoms. The finding that conduct disorder problems and hyperactivity/inattention were more pronounced in younger children supports pre-pandemic data that externalising problems are usually higher in younger children compared to older children (Lawrence et al., 2015). However, the findings that age did not predict greater levels of internalising symptoms particularly depressive symptoms - is surprising, given that a known risk for the onset of these mental health problems in adolescence (Kessler et al., 2005). It may be that increases in family stress, caused by the demands of balancing homeschooling and work may have been a particular challenge for parents of younger children. Indeed, younger children would have been more reliant on their parents for educational support and other provision of needs throughout the day. This result is consistent with findings from the UK that preadolescent children tended to experience greater deterioration in mental health symptoms, including emotional symptoms, whereas adolescents' emotional symptoms improved over the first month of lockdown (Waite et al., 2021), perhaps due to an ability to better maintain peer relationships via the use of technology (Li et al., 2021). Longitudinal research investigating the influence of age effects, family stress and peer relationships on changes in mental health symptoms during the course of the pandemic is an important area for future research.

Children with a pre-existing mental health disorder, neurodevelopmental condition or chronic physical illness experienced higher levels of parent-reported mental health symptoms compared to those without these difficulties. This finding is consistent with other research $(\mathrm{Li}$ 
et al., 2021; Pellicano et al., 2021) and highlights the need for policymakers to expand existing mental health services to support these vulnerable groups of children. The impact of the pandemic on parents' employment status was not associated with parent-reported youth mental health symptoms; however, families who applied for government financial assistance during COVID-19 reported that their child experienced higher levels of conduct disorder problems and depressive symptoms. This highlights that financial hardship as a result of the pandemic is a risk factor for youth mental health and justifies the existence of financial assistance for families affected by the pandemic.

Parent mental health symptoms significantly predicted youth mental health symptoms. Research has shown that parental mental health has deteriorated over the course of the pandemic (Strum et al., 2021; Westrupp et al., 2021). Thus, guidance and support to help parents improve coping skills may assist both parents and their children. However, we acknowledge the possibility that the association between parent mental health symptoms and child mental health symptoms may be more representative of parents providing higher ratings of their child's symptoms in the context of their own symptoms (Becker-Haimes et al., 2017). Collecting youth-reported data would overcome this issue in future research.

Children with a close contact with COVID-19 experienced higher levels of conduct disorder problems, anxiety symptoms and depressive symptoms. This suggests that concerns about the health impacts of COVID-19 on family and friends were relevant for youth mental health. Children who asked more frequent questions about COVID-19 were reported to have higher levels of emotional symptoms, anxiety symptoms and depressive symptoms. This result is perhaps unsurprising; and shows that children who worried more were more likely to ask questions about COVID-19 (or seek reassurance about it). Nevertheless, this finding highlights that parent-reported youth internalising symptoms were, in part, related to the pandemic itself. 
In contrast to research outside of Australia (Barendse et al., 2021), severity of government restrictions nor self-isolation status was associated with youth mental health symptoms. Given $60 \%$ of the sample were in the highest stages of lockdown when completing the survey, this result is unlikely to reflect that participants were not in lockdown when completing the survey. It may reflect that the threat of the pandemic itself was a risk factor for mental health. Alternatively, it may be that the impact of restrictions on known risk factors may be more relevant for understanding how lockdown policies impact youth mental health.

This study has a few limitations. The study population was not a representative sample and there was a bias towards more affluent families with Oceanian backgrounds. Given the high levels of mental health symptoms in low-income households within our study, we expect that the levels of difficulties reported here are an underestimation of the extent of difficulties experienced by the broader Australian community. We used an outcome measure (RCADS25-P) which has not been validated for young children (Years K to 2; aged 4 to 7 years). Results were similar when we excluded these children; however, future research might benefit from including a measure for anxiety and depressive symptoms developed and validated for younger children (e.g., PAS; Spence et al., 2001). Finally, while this study examined commonly occurring mental health symptoms for youth using predominantly wellvalidated screening measures that are relatively easy and speedy to administer, we did not assess the presence of mental health disorders using diagnostic classifications. Research that incorporates diagnostic measures is required to determine the full impact of COVID-19 on mental health impairment in youth samples.

These findings have important implications. Expansion of youth mental health services is imperative - particularly for vulnerable groups - to mitigate the effects of the pandemic on youth mental health. A focus on the prevention of mental health difficulties in children and 
adolescents and providing young people with strategies to manage their mental health, particularly during times of crisis, is also necessary. Youth mental health services are already overburdened (Young, 2020), and despite a move to the use of telehealth, more needs to be done to increase efficiencies in the delivery of mental health services, for example through increased use of evidence-based digital interventions (e.g., March et al., 2008; Spence et al., 2011).

In conclusion, these findings indicate that parent-reported youth mental health symptoms are notably high during the initial stages of the pandemic in Australia. Mental health symptoms are greater in children with pre-existing mental health, neurodevelopmental or physical health difficulties, and for children whose parents have experienced greater psychological distress and financial hardship. Our findings highlight the need for targeted and adequate support for youth and their families during the pandemic to reduce the burden of mental health difficulties among Australian youth.

\section{Acknowledgements}

We thank Emily Daniel for assisting with collecting information, including her work developing the stage of lockdown variable.

\section{Funding}

GS was supported by a Macquarie University Research Fellowship for the duration of this project (MQIRGR0001041). EP was supported by an Australian Research Council Future Fellowship (FT190100077) for the duration of this project. SM, CD, VC, AR, JR and LU acknowledge funding provided by the Medical Research Future Fund through the Department of Health (APP1179490). 


\section{Conflicts of interest}

EP reports occasional fees for workshops and invited addresses, and grants from the Australian Research Council, Medical Research Future Fund (Australia), the Cooperative Research Centre for Living with Autism ('Autism CRC'; Australia), the National Health and Medical Research Council (Australia), Positive Partnerships (Australia), and the European Commission, outside the submitted work. 


\section{References}

Barendse M, Flannery JE, Cavanagh C, Aristizabal M, Becker SP, Berger E, ... Pfeifer JH (2021, February 3). Longitudinal change in adolescent depression and anxiety symptoms from before to during the COVID-19 pandemic: A collaborative of 12 samples from 3 countries. https://doi.org/10.31234/osf.io/hn7us

Becker-Haimes EM, Jensen-Doss A, Birmaher B, et al. (2017) Parent-youth informant disagreement: Implications for youth anxiety treatment. Clinical Child Psychology and Psychiatry 23(1): 42-56.

Bromfield N and McConnell A (2020) Two routes to precarious success: Australia, New Zealand, COVID-19 and the politics of crisis governance. International Review of Administrative Sciences. DOI: 10.1177/0020852320972465. 0020852320972465.

Bubonya M, Cobb-Clark DA and Wooden M (2017) Job loss and the mental health of spouses and adolescent children. IZA Journal of Labor Economics 6(1): 6.

Ebesutani C, Korathu-Larson P, Nakamura BJ, et al. (2016) The Revised Child Anxiety and Depression Scale 25-Parent Version: Scale Development and Validation in a SchoolBased and Clinical Sample. Assessment 24(6): 712-728.

Goodman R (1997) The Strengths and Difficulties Questionnaire: a research note. J Child Psychol Psychiatry 38(5): 581-586.

Hansen BH, Oerbeck B, Skirbekk B, et al. (2018) Neurodevelopmental disorders: prevalence and comorbidity in children referred to mental health services. Nordic Journal of Psychiatry 72(4): 285-291.

Holmes EA, O'Connor RC, Perry VH, et al. (2020) Multidisciplinary research priorities for the COVID-19 pandemic: a call for action for mental health science. The Lancet Psychiatry 7(6): 547-560. 
Jiao WY, Wang LN, Liu J, et al. (2020) Behavioral and Emotional Disorders in Children during the COVID-19 Epidemic. The Journal of pediatrics 221: 264-266.e261.

Kessler RC, Berglund P, Demler O, et al. (2005) Lifetime prevalence and age-of-onset distributions of DSM-IV disorders in the National Comorbidity Survey Replication. Arch Gen Psychiatry 62(6): 593-602.

Lawrence D, Johnson S, Hafekost J, et al. (2015) The mental health of children and adolescents: Report on the second Australian child and adolescent survey of mental health and wellbeing.

Lawrence PJ, Murayama K and Creswell C (2019) Systematic Review and Meta-Analysis: Anxiety and Depressive Disorders in Offspring of Parents With Anxiety Disorders. Journal of the American Academy of Child and Adolescent Psychiatry 58(1): 46-60.

Li SH, Beames JR, Newby JM, et al. (2021) The impact of COVID-19 on the lives and mental health of Australian adolescents. European Child \& Adolescent Psychiatry. DOI: $10.1007 / \mathrm{s} 00787-021-01790-\mathrm{x}$.

Loades ME, Chatburn E, Higson-Sweeney N, et al. (2020) Rapid Systematic Review: The Impact of Social Isolation and Loneliness on the Mental Health of Children and Adolescents in the Context of COVID-19. Journal of the American Academy of Child and Adolescent Psychiatry 59(11): 1218-1239.e1213.

Lovibond PF and Lovibond SH (1995) The structure of negative emotional states: Comparison of the Depression Anxiety Stress Scales (DASS) with the Beck Depression and Anxiety Inventories. Behaviour Research and Therapy 33(3): 335343.

Magson NR, Freeman JYA, Rapee RM, et al. (2021) Risk and Protective Factors for Prospective Changes in Adolescent Mental Health during the COVID-19 Pandemic. Journal of Youth and Adolescence 50(1): 44-57. 
March S, Spence SH and Donovan CL (2008) The Efficacy of an Internet-Based CognitiveBehavioral Therapy Intervention for Child Anxiety Disorders. Journal of Pediatric Psychology 34(5): 474-487.

Nguyen J, Hinojosa MS, Strickhouser Vega S, et al. (2017) Family Predictors of Child Mental Health Conditions. Journal of Family Issues 39(4): 935-959.

Orben A, Tomova L and Blakemore S-J (2020) The effects of social deprivation on adolescent development and mental health. The Lancet Child \& Adolescent Health 4(8): 634-640.

Pellicano E, Brett S, den Houting J, Heyworth M, Magiati I, Steward R, Urbanowicz A and Stears M. COVID-19, Social Isolation and the Mental Health of Autistic People and Their Families: A Qualitative Study. http://dx.doi.org/10.2139/ssrn.3749228

Pearcey S, Shum A, Waite P, Patalay P and Creswell C (2020, June 16). Report 04: Changes in children and young people's emotional and behavioural difficulties through lockdown. http://cospaceoxford.org/wp-content/uploads/2020/07/CoSPACE-Report4-June-2020.pdf

Perneger TV (1998) What's wrong with Bonferroni adjustments. BMJ 316(7139): 1236-1238. Pfeffer M (2021) Covid-19 and compliance: What makes people stick to the rules? In ANZSOG 2021. Available at: https://www.anzsog.edu.au/resource-library/newsmedia/covid-19-and-compliance (accessed 30 May 2021).

Pinquart M and Shen Y (2011a) Behavior problems in children and adolescents with chronic physical illness: a meta-analysis. J Pediatr Psychol 36(9): 1003-1016.

Pinquart M and Shen Y (2011b) Depressive symptoms in children and adolescents with chronic physical illness: an updated meta-analysis. J Pediatr Psychol 36(4): 375-384.

Racine N, Cooke JE, Eirich R, et al. (2020) Child and adolescent mental illness during COVID-19: A rapid review. Psychiatry research 292: 113307-113307. 
Raw J, Waite P, Pearcey S, Creswell C, Shum A, and Patalay P (2021, February 3).

Examining changes in parent-reported child and adolescent mental health throughout the UK's first COVID-19 national lockdown. https://doi.org/10.31234/osf.io/exktj

Rosen Z, Weinberger-Litman SL, Rosenzweig C, Rosmarin DH, Muennig P, Carmody ER, ... Litman L (2020, April 14). Anxiety and distress among the first community quarantined in the U.S due to COVID-19: Psychological implications for the unfolding crisis. https://doi.org/10.31234/osf.io/7eq8c

Salbach-Andrae H, Klinkowski N, Lenz K, et al. (2009) Agreement between youth-reported and parent-reported psychopathology in a referred sample. Eur Child Adolesc Psychiatry 18(3): 136-143.

Seward RJ, Bayliss DM, Stallman HM, et al. (2018) Psychometric Properties and Norms for the Strengths and Difficulties Questionnaire Administered Online in an Australian Sample. Australian Psychologist 53(2): 116-124.

Shum A, Skripkauskaite S, Pearcey S, Raw J, Waite P, Creswell, C (2021, January 19). Report 07: Changes in parents' mental health symptoms and stressors from April to December 2020. http://cospaceoxford.org/wp content/uploads/2021/01/Report 07_19JAN-1.pdf

Spence SH, Donovan CL, March S, et al. (2011) A randomized controlled trial of online versus clinic-based CBT for adolescent anxiety. Journal of Consulting and Clinical Psychology 79(5): 629-642.

Spence SH, Rapee R, McDonald C, et al. (2001) The structure of anxiety symptoms among preschoolers. Behaviour Research and Therapy 39(11): 1293-1316.

Waite P, Pearcey S, Shum A, et al. (2021) How did the mental health symptoms of children and adolescents change over early lockdown during the COVID-19 pandemic in the UK? JCPP Advances 1(1): e12009. 
Waite P, Creswell C (2020) Co-SPACE (Covid-19: Supporting Parents, Adolescents and Children during Epidemics. https://osf.io/8zx2y/

Westrupp E, Bennett C, Berkowitz TS, Youssef GJ, Toumbourou J, Tucker R, ... Sciberras E (2020, September 30). Child, parent, and family mental health and functioning in Australia during COVID-19: Comparison to pre-pandemic data. https://doi.org/10.31234/osf.io/ydrm9

Whittle S., Bray KO, Lin S, and Schwartz O (2020, August 5). Parenting and child and adolescent mental health during the COVID-19 pandemic. https://doi.org/10.31234/osf.io/ag2r7

Wickham S, Whitehead M, Taylor-Robinson D, et al. (2017) The effect of a transition into poverty on child and maternal mental health: a longitudinal analysis of the UK Millennium Cohort Study. The Lancet Public Health 2(3): e141-e148.

World Health Organisation (2021) WHO Coronovirus Disease (COVID-9) dashboard. World Health Organization. https://covid19.who.int/ Published 2021. Accessed 04 May 2020.

Xie X, Xue Q, Zhou Y, et al. (2020) Mental Health Status Among Children in Home Confinement During the Coronavirus Disease 2019 Outbreak in Hubei Province, China. JAMA Pediatrics 174(9): 898-900.

Young E (2020) Coronavirus worries have Australian children calling Kids Helpline every 69 seconds. In: SBS News 2020. Available at: https://www.sbs.com.au/news/coronavirus-worries-have-australian-children-callingkids-helpline-every-69-seconds (accessed 01 Aug 2020).

Zubrick S, Silburn SR, Lawrence D, Mitrou FG, Dalby RB, Blair E, Griffin J, Milroy H, De Maio JA, Cox A and Li, J (2005). The Western Australian Aboriginal Child Health Survey: The social and emotional wellbeing of Aboriginal children and young people. 
vol. 2, Curtin University of Technology and the Telethon Institute for Child Health Research, Perth, Western Australia. 
Table 1 . Sample characteristics, by age group and total sample

\begin{tabular}{|c|c|c|c|}
\hline Sociodemographic and background factors & $\begin{array}{l}\text { Children (4-11 years) } \\
\qquad(n=791)\end{array}$ & $\begin{array}{l}\text { Adolescents (12-17 years) } \\
\qquad(n=536)\end{array}$ & Whole sample $(\mathrm{n}=1327)$ \\
\hline \multicolumn{4}{|l|}{ Parent gender, n (\%) } \\
\hline Female & $564(71.4)$ & $364(67.9)$ & $928(69.9)$ \\
\hline Other & $2(0.2)$ & $2(0.4)$ & $4(0.4)$ \\
\hline \multicolumn{4}{|l|}{ Parent ethnicity, n (\%) } \\
\hline Oceanian (e.g., Australian, New Zealander) & $474(60.2)$ & $308(57.8)$ & $782(58.9)$ \\
\hline North African and Middle Eastern & $8(1.0)$ & $1(0.2)$ & $9(0.7)$ \\
\hline South East Asian & $51(6.5)$ & $27(5.1)$ & $78(5.9)$ \\
\hline North East Asian & $22(2.8)$ & $23(4.1)$ & $45(3.4)$ \\
\hline Southern and Central Asian & $58(7.4)$ & $22(4.1)$ & $80(6.0)$ \\
\hline
\end{tabular}


MENTAL HEALTH OF AUSTRALIAN YOUTH DURING COVID-19 29

\begin{tabular}{|c|c|c|c|}
\hline Peoples of the Americas & $10(1.3)$ & $6(1.1)$ & $16(1.2)$ \\
\hline \multicolumn{4}{|c|}{ Parent Aboriginal or Torres Strait Islander origin, $\mathrm{n}(\%)$} \\
\hline Yes & $64(8.1)$ & $29(5.5)$ & $93(7.0)$ \\
\hline \multicolumn{4}{|l|}{ Parent education, $\mathrm{n}(\%)$} \\
\hline School/vocational qualifications & $247(31.2)$ & $211(39.4)$ & $458(34.5)$ \\
\hline Undergraduate degree & $344(43.5)$ & $200(37.4)$ & $544(41.0)$ \\
\hline Not employed/studying & $154(19.5)$ & $92(17.2)$ & $246(18.5)$ \\
\hline Child age, $M(\mathrm{SD})$ & $7.7(2.2)$ & $14.5(1.7)$ & $10.4(3.9)$ \\
\hline \multicolumn{4}{|l|}{ Child gender } \\
\hline Male & $424(53.9)$ & $288(53.9)$ & $712(53.7)$ \\
\hline Female & $359(45.6)$ & $244(45.7)$ & $603(45.4)$ \\
\hline
\end{tabular}




\begin{tabular}{|c|c|c|c|}
\hline Other & $4(0.5)$ & $2(0.4)$ & $6(0.5)$ \\
\hline \multicolumn{4}{|l|}{ Child ethnicity, n (\%) } \\
\hline Oceanian (e.g., Australian, New Zealander) & $474(60.2)$ & $308(57.8)$ & $821(61.9)$ \\
\hline North-West European (e.g., British) & $106(13.5)$ & $105(19.7)$ & $186(14.0)$ \\
\hline Southern and Eastern European & $53(6.7)$ & $38(7.1)$ & $82(6.2)$ \\
\hline North African and Middle Eastern & $8(1.0)$ & $1(0.2)$ & $9(0.7)$ \\
\hline South East Asian & $51(6.5)$ & $27(5.1)$ & $79(6.0)$ \\
\hline North East Asian & $22(2.8)$ & $23(4.3)$ & $45(3.4)$ \\
\hline Southern and Central Asian & $58(7.4)$ & $22(4.1)$ & $75(5.7)$ \\
\hline Peoples of the Americas & $10(1.3)$ & $6(1.1)$ & $13(1.0)$ \\
\hline Sub-Saharan African & $6(0.8)$ & $3(0.6)$ & $11(0.8)$ \\
\hline \multicolumn{4}{|c|}{ Child Aboriginal or Torres Strait Islander origin, $\mathrm{n}(\%)$} \\
\hline Yes & $73(9.3)$ & $33(6.2)$ & $106(8.0)$ \\
\hline No & $714(90.7)$ & $500(93.8)$ & $1214(91.5)$ \\
\hline \multicolumn{4}{|l|}{ Child's education type, $\mathrm{n}(\%)$} \\
\hline Public school & $518(66.1)$ & $334(62.5)$ & $852(64.6)$ \\
\hline
\end{tabular}


MENTAL HEALTH OF AUSTRALIAN YOUTH DURING COVID-19 31

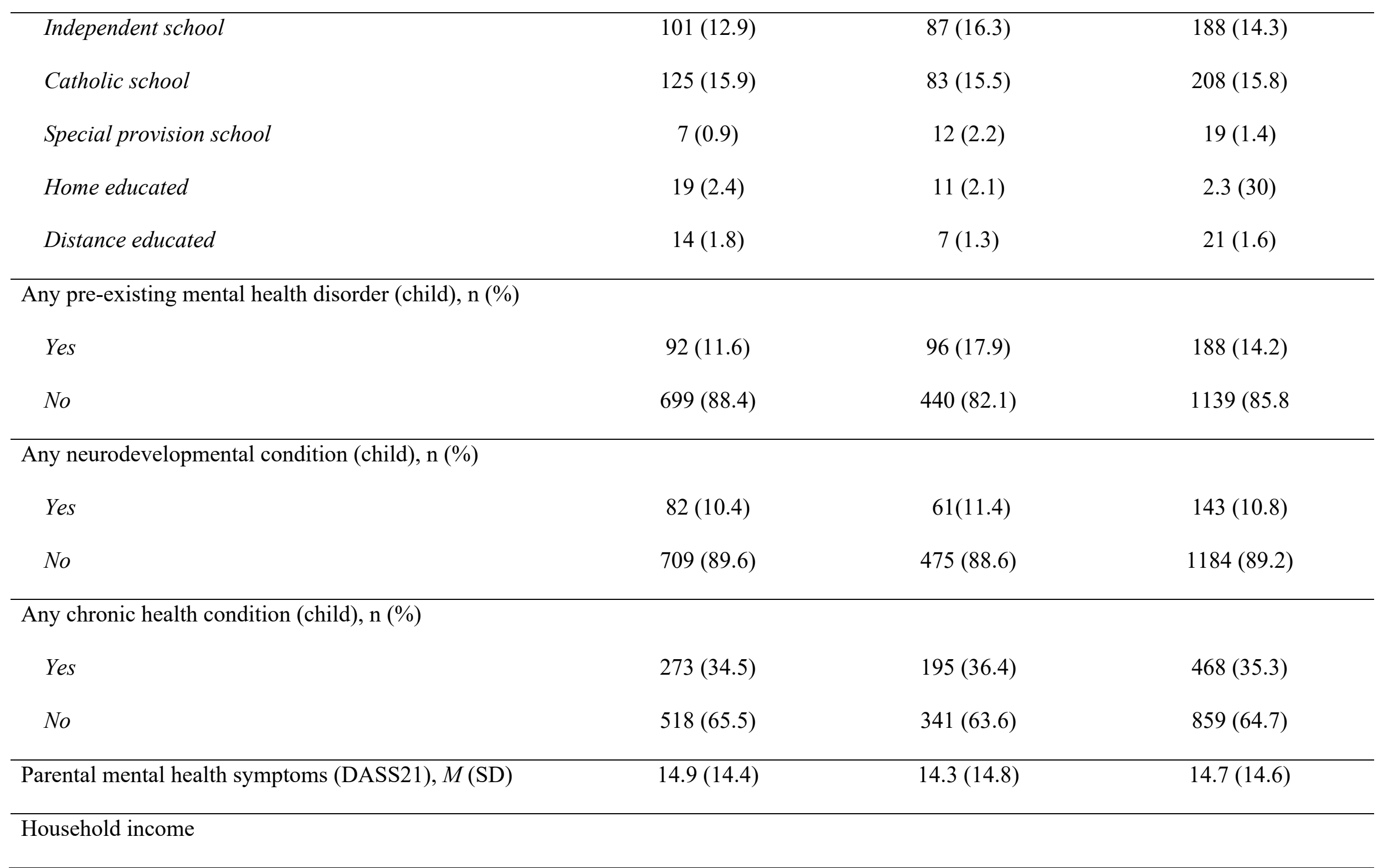


MENTAL HEALTH OF AUSTRALIAN YOUTH DURING COVID-19 32

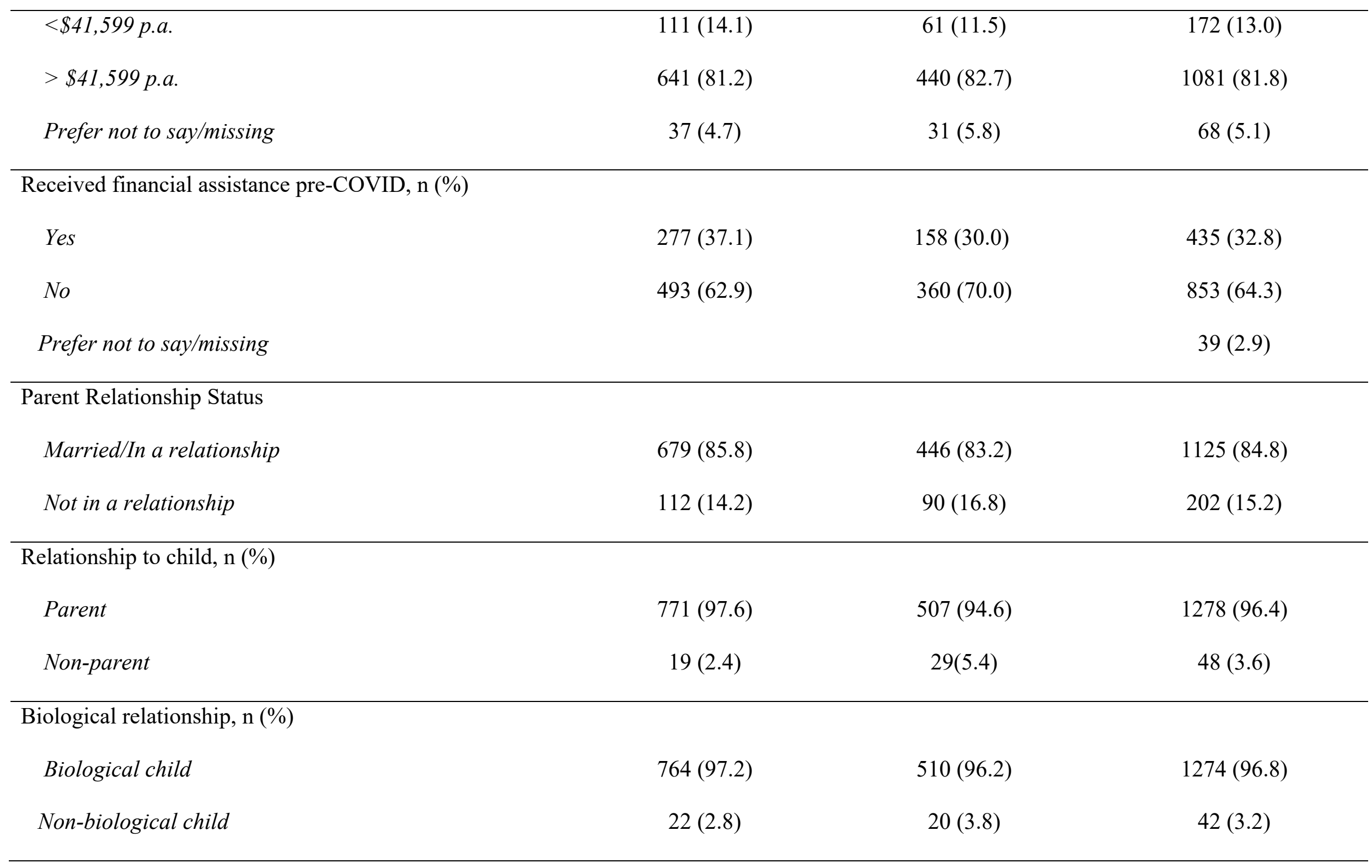




\begin{tabular}{|c|c|c|c|}
\hline \multicolumn{4}{|l|}{ Geographic state, n (\%) } \\
\hline$N S W$ & $168(21.2)$ & $107(20.0)$ & $275(20.7 \%)$ \\
\hline$V I C$ & $356(45.0)$ & $225(42.0)$ & $581(43.8 \%)$ \\
\hline$A C T$ & $4(0.5)$ & $3(0.6)$ & $7(0.5 \%)$ \\
\hline$N T$ & $5(0.6)$ & $1(0.2)$ & $6(0.5 \%)$ \\
\hline$S A$ & $31(3.9)$ & $22(4.1)$ & $53(4.0 \%)$ \\
\hline$T A S$ & $9(1.1)$ & $10(1.9)$ & $19(1.4 \%)$ \\
\hline Non-Major Cities of Australia & $132(16.8)$ & $88(16.7)$ & $220(16.8)$ \\
\hline \multicolumn{4}{|l|}{ COVID-19 exposure } \\
\hline \multicolumn{4}{|l|}{ Child has COVID-19, n (\%) } \\
\hline Yes, diagnosed or suspected & $71(9.0)$ & $32(6.0)$ & $103(7.8)$ \\
\hline No & $718(91.0)$ & $503(94.0)$ & $1221(92.2)$ \\
\hline
\end{tabular}




\begin{tabular}{|c|c|c|c|}
\hline \multicolumn{4}{|l|}{ Close contact has COVID-19, n (\%) } \\
\hline \multicolumn{4}{|l|}{ COVID-19 Impact \& Measures } \\
\hline Yes & $266(33.6)$ & $173(32.3)$ & $439(33.1)$ \\
\hline No & $525(66.4)$ & $363(67.7)$ & $888(66.9)$ \\
\hline \multicolumn{4}{|l|}{ Social isolation status, $\mathrm{n}(\%)$} \\
\hline Living life as normal/not strictly self-isolating & $605(76.7)$ & $410(76.9)$ & $1015(76.8)$ \\
\hline \multicolumn{4}{|l|}{$(\%)$} \\
\hline Yes & $207(28.7)$ & $129(26.4)$ & $336(26.9)$ \\
\hline No & $464(64.3)$ & $332(68.0)$ & $796(63.7)$ \\
\hline Not eligible/missing & & & $117(9.4)$ \\
\hline Child asking questions about COVID-19, $M$ (SD) & $3.8(0.8)$ & $3.8(0.8)$ & $3.8(0.8)$ \\
\hline
\end{tabular}




\begin{tabular}{|c|c|c|c|}
\hline Time since onset of pandemic, $M$ days (SD) & $126.7(50.5)$ & $125.6(47.2)$ & $126.2(49.0)$ \\
\hline Stage 2 & $30(3.8)$ & $44(8.2)$ & $74(5.6)$ \\
\hline Stage 3 & $255(32.3)$ & $173(32.3)$ & $428(32.3)$ \\
\hline Stage 5 & $191(24.2)$ & $114(21.3)$ & $305(23.0)$ \\
\hline \multicolumn{4}{|l|}{ Mental health outcome measures } \\
\hline SDQ Emotional Symptoms, $M$ score (SD) & $3.2(2.7)$ & $3.0(2.8)$ & $3.1(2.7)$ \\
\hline RCADS25, Anxiety, $M$ score, score (SD) & $9.7(8.6)$ & $8.9(8.9)$ & $9.4(8.8)$ \\
\hline RCADS25 Depression, $M$ score (SD) ${ }^{*}$ & $5.7(6.0)$ & $6.2(6.4)$ & $5.9(6.2)$ \\
\hline
\end{tabular}

Note. ${ }^{*}$ indicates statistically significant difference between two independent (age) groups on mental health outcome measures at $p<.05$. 


\section{MENTAL HEALTH OF AUSTRALIAN YOUTH DURING COVID-19 36}

Table 2. Prevalence of symptoms based on parent-report subscales of the SDQ and RCADS25, by age group and total sample

\begin{tabular}{|c|c|c|c|c|c|c|c|c|c|}
\hline & \multicolumn{3}{|c|}{ Children (4-11 years) } & \multicolumn{4}{|c|}{ Adolescents (12-17 years) } & \multicolumn{2}{|c|}{ Total sample } \\
\hline & $\begin{array}{l}\text { Existing } \\
\text { diagnosis }\end{array}$ & $\begin{array}{l}\text { No } \\
\text { diagnosis }\end{array}$ & $\begin{array}{l}\text { Total } \\
\text { sample }\end{array}$ & $\begin{array}{l}\text { Existing } \\
\text { diagnosis }\end{array}$ & $\begin{array}{l}\text { No } \\
\text { diagnosis }\end{array}$ & $\begin{array}{l}\text { Total } \\
\text { Sample }\end{array}$ & $\begin{array}{l}\text { Existing } \\
\text { diagnosis }\end{array}$ & $\begin{array}{l}\text { No } \\
\text { diagnosis }\end{array}$ & Total Sample \\
\hline SDQ subscales sample & $n=92$ & $n=698$ & $n=790$ & $n=95$ & $n=440$ & $n=535$ & $n=187$ & $n=1138$ & $n=1325$ \\
\hline \multicolumn{10}{|c|}{ Elevated Emotional Symptoms, n (\%) } \\
\hline Slightly raised & $12(13.0)$ & $72(10.3)$ & $84(10.6)$ & $11(11.6)$ & $36(8.2)$ & $47(8.8)$ & $23(9.5)$ & $108(9.5)$ & $131(9.9)$ \\
\hline High/Very High & $53(57.6)$ & $183(26.2)$ & $236(29.9)$ & $62(65.3)$ & $106(24.2)$ & $168(31.4)$ & $115(61.5)$ & $289(25.4)$ & $404(30.5)$ \\
\hline \multicolumn{10}{|c|}{ Elevated Conduct Problems, n (\%) } \\
\hline Slightly raised & $18(19.6)$ & $69(9.9)$ & $87(11.0)$ & $13(13.7)$ & $35(8.0)$ & $48(9.0)$ & $31(16.6)$ & $104(9.1)$ & $135(10.2)$ \\
\hline High/Very High & $33(35.9)$ & $190(27.2)$ & $223(28.2)$ & $32(33.7)$ & $93(21.1)$ & $125(23.4)$ & $65(34.8)$ & $283(24.9)$ & $348(26.3)$ \\
\hline \multicolumn{10}{|c|}{ Elevated Hyperactivity Problems, n (\%) } \\
\hline Slightly raised & $21(22.8)$ & $130(18.6)$ & $151(19.1)$ & $22(23.2)$ & $67(15.2)$ & $89(16.6)$ & $43(23.0)$ & $197(17.3)$ & $240(18.1)$ \\
\hline High/Very High & $35(38.0)$ & $56(8.0)$ & $91(11.5)$ & $24(25.3)$ & $11(2.5)$ & $35(6.5)$ & $59(31.6)$ & $67(5.9)$ & $126(9.5)$ \\
\hline RCADS25-P subscale sample & $n=88$ & $n=694$ & $n=782$ & $n=95$ & $n=437$ & $n=532$ & $n=184$ & $n=1131$ & $n=1315$ \\
\hline
\end{tabular}


MENTAL HEALTH OF AUSTRALIAN YOUTH DURING COVID-19 37

Elevated Anxiety Symptoms, n (\%)

$\begin{array}{llllllllll}\text { Borderline } & 10(11.4) & 34(4.9) & 44(5.6) & 11(11.6) & 13(3.0) & 24(4.5) & 21(11.5) & 47(4.2) & 68(5.2) \\ \text { Clinical } & 30(34.1) & 118(17.0) & 148(18.9) & 34(35.8) & 86(19.7) & 120(22.6) & 64(35.0) & 204(18.0) & 268(20.2)\end{array}$

Elevated Depressive Symptoms, n (\%)

$\begin{array}{llllllllll}\text { Borderline } & 4(4.5) & 34(4.9) & 38(4.9) & 13(13.7) & 30(6.9) & 43(8.1) & 17(9.2) & 64(5.7) & 81(6.1) \\ \text { Clinical } & 36(40.4) & 123(17.7) & 159(20.3) & 42(44.2) & 70(16.0) & 112(21.1) & 78(42.4) & 193(17.1) & 271(20.4)\end{array}$


Table 3. Linear regression models for each SDQ subscale outcome

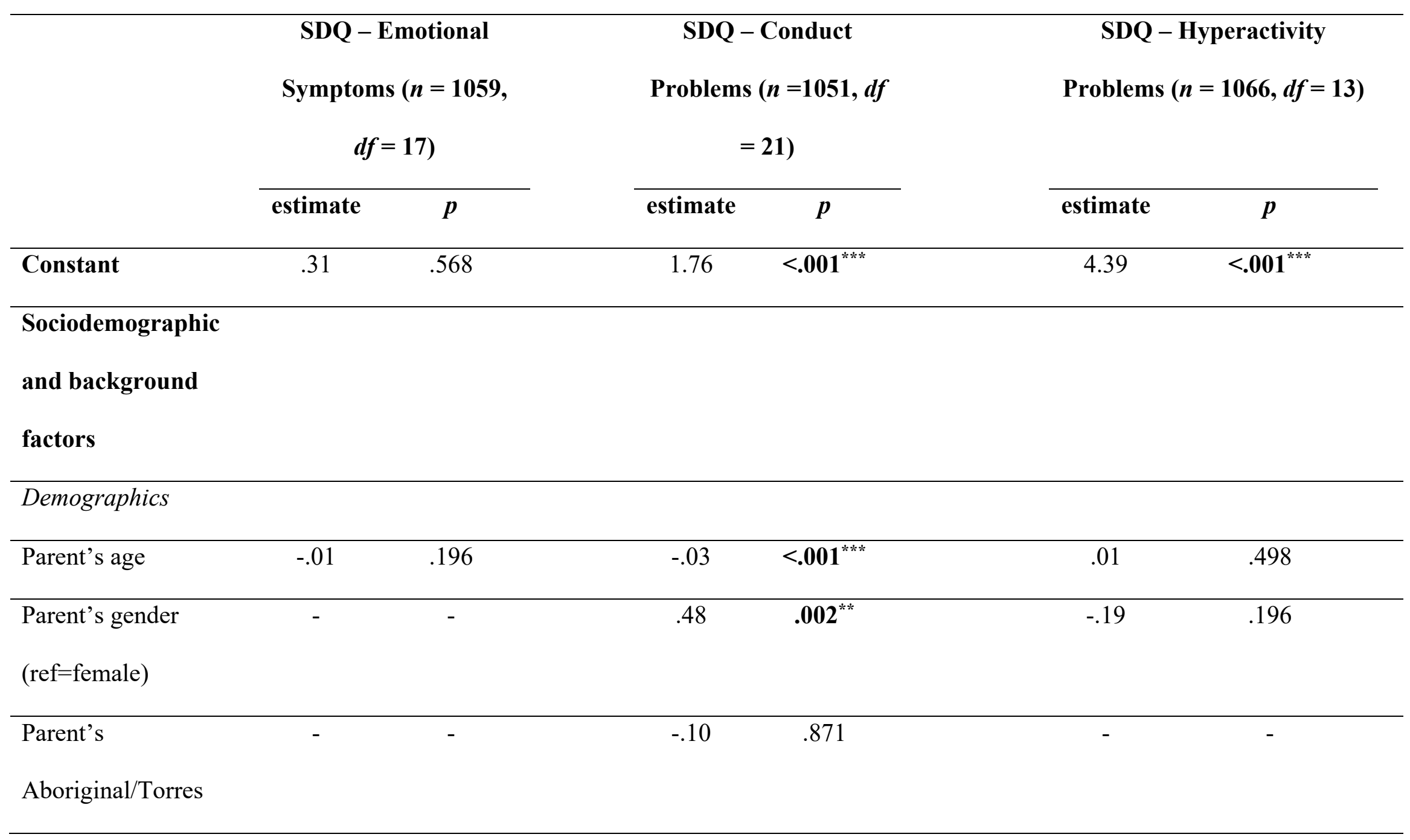


Strait Islander origin

status $(\mathrm{ref}=\mathrm{no})$

\begin{tabular}{lllllll} 
Child's age & - & - & -.03 & $\mathbf{. 0 4 5}^{*}$ & -.14 & $<$.001 $^{* * *}$ \\
\hline Child's gender (ref $=$ & -.43 & $\mathbf{. 0 0 2}^{* *}$ & - & - & .65 & $<.001^{* * *}$ \\
female) & & & & & &
\end{tabular}

Child's

$\begin{array}{lll}- & .31 & .577\end{array}$

Aboriginal/Torres

Strait Islander origin

status $(\mathrm{ref}=\mathrm{no})$

Child's education

.25

.438

$-.51$

.09

type (ref=public

school vs not)

Geographic location

.14

.472

$-$

(ref=Major Cities of

Australia) 
Child clinical/health

background

Any mental health

2.13

$<.001^{* * * *}$

.41

$.029^{*}$

$1.30<.001^{* * *}$

condition $($ ref $=$ no $)$

Any

.29

.272

.71

$.001^{* *}$

1.20

$<.001^{* * *}$

neurodevelopmental

condition $(\mathrm{ref}=\mathrm{no})$

Any chronic health

.25

.109

.04

.775

.27

.061

condition $(\mathrm{ref}=\mathrm{no})$

\section{Family}

factors/functioning

Parent mental health

.07

$<.001^{* * *}$

.06

$<.001^{* * *}$

$.04<.001^{* * *}$

symptoms (DASS21)

Household income

.14

.518

.05

.767

.03

.887

(ref=low income) 
Received government

.558

.02

.890

.26

.102

financial assistance

pre-COVID19

$(\mathrm{ref}=\mathrm{no})$

Relationship factors

Parent's relationship

.24

.240

-

$-.01$

.837

status (ref=married/in

a relationship)

Relationship to child

.68

.121

(ref=parent)

\section{Biological}

.68

.083

$-.46$

.277

relationship to parent

(ref=biological child)

\section{COVID-19 exposure}


Child suspected or .15

.638

$-.124$

.638

.18

.552

diagnosed COVID19

$(\mathrm{ref}=\mathrm{no})$

Close contact .36

.516

1.39

$.004^{* *}$

$-.84$

.100

diagnosed COVID19

$(\mathrm{ref}=\mathrm{no})$

\section{COVID-19 Impact}

\& Measures

Change in .25

.106

.01

.951

$-.02$

.877

employment status

$(\mathrm{ref}=\mathrm{no})$

Applied for .31

$.013^{*}$

.00

.970

.13

.288

government

assistance during

COVID-19 (ref=no) 
questions about

\section{COVID19}

(frequency)

Self-isolation status $-.09$ .602 $-.18$ .192

(ref=not strictly self-

isolating versus

strictly self-isolating)

Time since pandemic

$.01<.001^{*}$

declared

\begin{tabular}{|c|c|c|c|c|c|c|c|c|c|}
\hline & $R^{2}$ & Adjusted $R^{2}$ & $F$ & $R^{2}$ & Adjusted $R^{2}$ & $F$ & $R^{2}$ & $\begin{array}{c}\text { Adjusted } \\
R^{2}\end{array}$ & $F$ \\
\hline Model & .36 & .35 & $32.73^{* * *}$ & .33 & .31 & $24.77^{* * * *}$ & .24 & .23 & $19.34^{* * *}$ \\
\hline
\end{tabular}

${ }^{*} p<.05 ;{ }^{* *} p<.010,{ }^{* * *} p<.001$. Bold indicates tests significant at $p<.05$. 
Table 4. Linear regression models for each RCADS25P subscale outcome

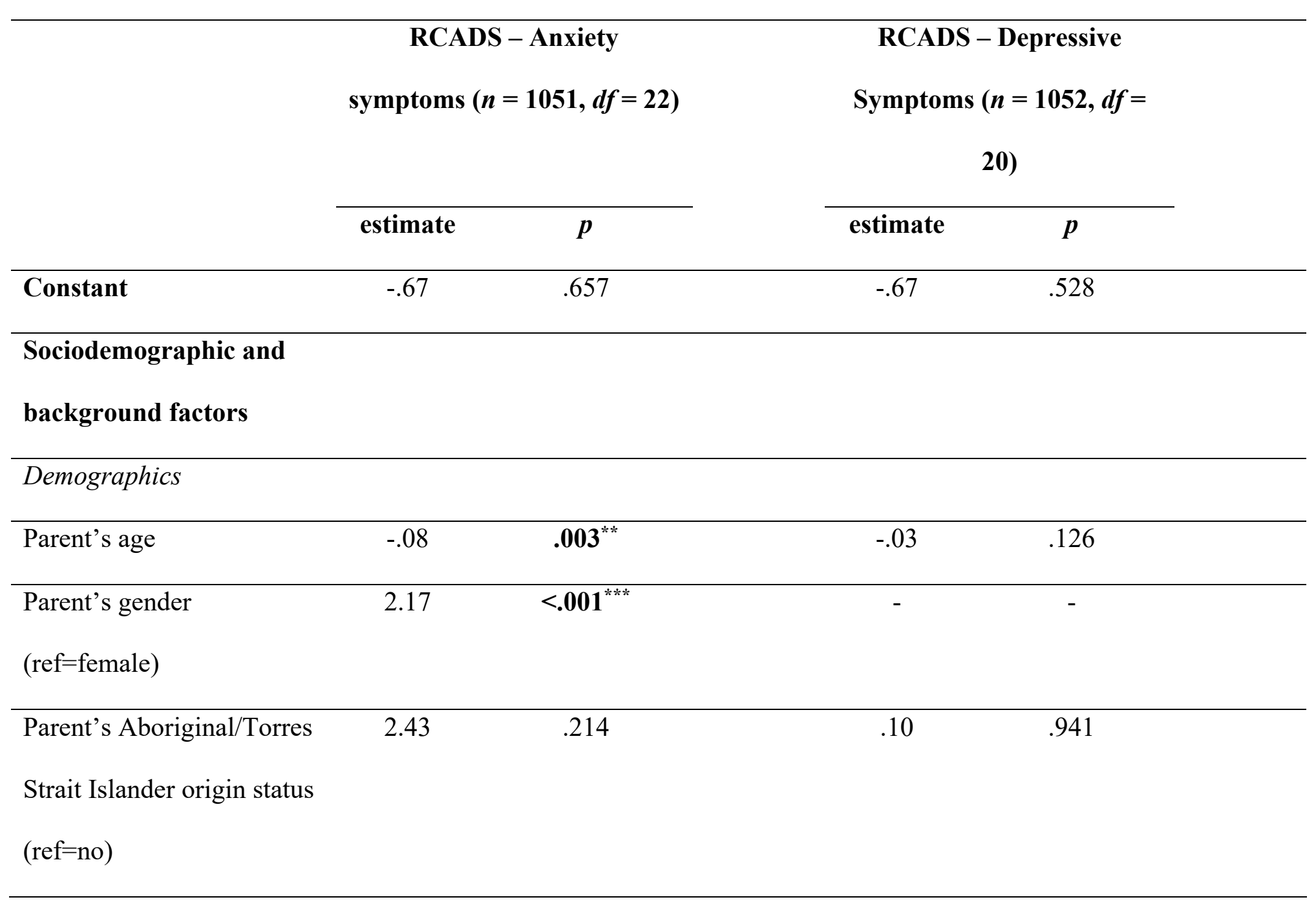




\begin{tabular}{lllll}
\hline Parent's employment status & -.86 & .113 & - & \\
(ref=employed vs not & & & \\
employed) & & & - & - \\
\hline Child's age & -.06 & .279 & -43 & .120 \\
\hline Child's gender (ref $=$ & -1.28 & $\mathbf{. 0 0 1}^{* * *}$ & \\
female) & & & -.38 \\
\hline Child's Aboriginal/Torres & -1.94 & .297 & \\
Strait Islander origin status & & & \\
(ref=no) & & .552 & .09 \\
\hline Child's education type & .52 & & \\
(ref=public school vs not) & & & \\
\hline Child clinical/health & & & \\
background & & & \\
\hline Any mental health & & & \\
condition (ref=no) & & & \\
\hline
\end{tabular}


Any neurodevelopmental

1.57

$.028^{*}$

1.34

.008 *

condition $(\mathrm{ref}=\mathrm{no})$

Any chronic health

1.07

$.012 *$

.88

$\mathbf{0 0 5}^{* *}$

condition $(\mathrm{ref}=\mathrm{no})$

Family factors/functioning

Parent mental health

.29

$<.001^{* * *}$

.22

$<.001^{* * *}$

symptoms (DASS21)

Household income

$-.10$

.865

$-.17$

.682

(ref=low income)

\section{Received government}

.89

.051

.23

.481

financial assistance pre-

COVID19 $(\mathrm{ref}=\mathrm{no})$

Relationship factors

Parent's relationship status

.46

.415

.71

.637

$(\mathrm{ref}=\mathrm{married} / \mathrm{in} \mathrm{a}$

relationship) 


\begin{tabular}{lllll}
\hline Relationship to child & .46 & .747 & .49 & .637 \\
(ref=parent) & & & \\
\hline Biological relationship to & 1.48 & .285 & 2.44 & $.014^{*}$ \\
parent (ref=biological & & & & \\
child) & & &
\end{tabular}

\section{COVID-19 exposure}

\begin{tabular}{lllll}
\hline Child suspected or & .06 & .947 & .34 & .592
\end{tabular}

diagnosed COVID19

$(\mathrm{ref}=\mathrm{no})$

Close contact diagnosed

6.11

$<.001$ *

4.03

$<.001 *$

COVID19 (ref=no)

\section{COVID-19 Impact \&}

\section{Measures}

Change in employment

.72

.087

.50

.095

status $(\mathrm{ref}=\mathrm{no})$ 
Applied for government

.61

.077

.52

$.033^{*}$

assistance during COVID-

$19(\mathrm{ref}=\mathrm{no})$

Child asking questions

2.06

$<.001^{* * *}$

.88

$<.001^{* * *}$

about COVID19

(frequency)

Self-isolation status

.18

.699

$-.13$

.701

(ref=not strictly self-

isolating versus strictly

self-isolating)

\begin{tabular}{lcccccc} 
& $\boldsymbol{R}^{2}$ & Adjusted $\boldsymbol{R}^{2}$ & $\boldsymbol{F}$ & $\boldsymbol{R}^{2}$ & Adjusted $\boldsymbol{R}^{2}$ & $\boldsymbol{F}$ \\
\hline Model & .54 & .53 & $\mathbf{5 2 . 3 4 ^ { * * * }}$ & .52 & .52 & $\mathbf{5 6 . 0 4}^{* * *}$ \\
\hline
\end{tabular}

${ }^{*} p<.01 ; * * * p<.001, * * * p<.001$. Bold indicates tests significant at $p<.01$. 